THE ASTROPHYSICAL JOURNAL, 304:305-311, 1986 May 1

C 1986. The American Astronomical Society. All rights reserved. Printed in U.S.A.

\title{
BV SURFACE PHOTOMETRY OF SOUTHERN GALAXIES. I. NGC 4507
}

\author{
T. Storchi Bergmann and M. G. Pastoriza \\ Instituto de Física, Universidade Federal do Rio Grande do Sul \\ Received 1985 February 14; accepted 1985 October 15
}

\begin{abstract}
From $B$ and $V$ photographic surface photometry we have obtained photometric parameters, meanluminosity profiles, and $B-V$ color indices for the barred spiral galaxy NGC 4507. The decomposition of the mean-luminosity $B$ profile shows the existence of an $r^{1 / 4}$ bulge, an exponential disk, a bar, and possibly a lens. The dimensions and colors of these structures are derived. The mean $B-V$ distribution shows a color gradient of $0.4 \mathrm{mag}$ between the nucleus and arms region. A reddening of $E(B-V)=0.2-0.3$ of the southern region can be detected when we compare its $B-V$ values with those of the northern region.
\end{abstract}

Subject headings: galaxies: individual - galaxies: photometry - galaxies: structure

\section{INTRODUCTION}

This work is part of a more extensive one whose primary aim is to make a comparative study of the structural and photometric parameters in the $B V$ system for a group of different morphological-type galaxies in order to obtain a more accurate information about the several components and stellar population.

This paper presents some results obtained for the spiral galaxy NGC 4507, classified as SBab(rs) I (Sandage and Brucato 1979). This galaxy has been previously classified in the Second Reference Catalogue (de Vaucouleurs, de Vaucouleurs, and Corwin 1976, hereafter $\mathrm{RC} 2$ ) as $\mathrm{SAB}^{+}$perhaps because of its small angular diameter $\left(\sim 2^{\prime}\right)$ and the bright, well-developed arms tightly warped around the nuclear region, which give it an appearance of a compact object in a small-scale photographic plate. Combining short, intermediate, and long-exposure photographic plates, the nuclear region is resolved in a very bright stellar nucleus and diffuse asymmetric bar, and numerous bright $\mathrm{H}$ II regions are observed in the spiral arms.

Photoelectric observations of this galaxy were made by Wegner (1979) and Griersmith (1980). Its radial velocity (3540 $\mathrm{km} \mathrm{s}^{-1}$ ) was measured by Martin (1976).

\section{OBSERVATIONS AND REDUCTIONS}

Five direct photographic plates were obtained with the Cassegrain camera attached to the $1.6 \mathrm{~m}$ telescope of Observatorio Astrofísico Brasileiro. A combination of IIa-O or $103 \mathrm{a}-\mathrm{O}$ plate plus GG 385 filter was used for the $B$ system and a combination of IIa-D or 103 a-D plus GG 495 for the $V$ system. Details of the observations are listed on Table 1 . The scale of the plates is $13^{\prime \prime} \mathrm{mm}^{-1}$. The calibration was made with a spot sensitometer.

The intermediate exposure plate presented a deflect and could not be used for photometric measurements. The other four plates were scanned with the PDS microdensitometer of Observatorio Nacional, using a diaphragm of $50 \times 50 \mu \mathrm{m}$ and steps in $x$ and $y$ of the same size. The reduction was made using the software developed by da Costa et al. (1982). The main steps are described below.

The density measures were converted to intensities by means of the de Vaucouleurs relation:

$$
\log E=\sum_{n=0}^{M} A_{n}(\log w)^{n},
$$

where $w=10^{(d-d f)}-1, d$ is the density of the calibration spot, and $d f$ is the density of the fog. The sky background was then determined as the mean from four selected areas around the galaxy. The differences between the mean values for each area were smaller than the internal fluctuation within the same area. This means that the sky background around the galaxy is approximately uniform, justifying the adopted procedure. The sky intensity was subtracted from the image matrix, and, in order to obtain the magnitude scale, circular apertures were simulated and the instrumental magnitudes within them compared with photoelectric $B V$ data from Wegner (1979) and Griersmith (1980). After the calibration, the mean sky magnitude obtained for the plate No. 104, on which most of the results and conclusions rely, was $B=21.75 \pm 0.05$.

The short-exposure plates were used to define the region within $5^{\prime \prime}$ from the nucleus. The short- and long-exposure average profiles between $2^{\prime \prime}$ and $5^{\prime \prime}$ overlap, with a mean difference of $0.08 \mathrm{mag}$. Farther than $5^{\prime \prime}$, the agreement is poorer because the short-exposure-profiles become too faint. The seeing was estimated by the fit of a Gaussian function $s(x)=A$ $\exp \left(-x^{2} / 2 \sigma^{2}\right)$ to stellar images in the plates. For the longexposure plates we obtained $\sigma \approx 1^{\prime \prime}$, but for the short-exposure ones we obtained $\sigma \approx 0$ ". 6 . The smoothing effect of the seeing is more important in the central peak. Considering that the seeing was very small for the short-exposure plates (from which the central data was obtained), we neglected its effect.

The accuracy of the data was checked using the surface photometry obtained by one of the authors (Schröder and Pastoriza 1985, hereafter SP) for the galaxy NGC 2855. The reduction procedure was the same adopted here. Figure 1 shows the difference between the elliptically average $B$ profile obtained by SP and the one from Boroson (1981). The fact that in the inner region the values of SP are systematically brighter than that of Boroson agrees with the results obtained by Whitmore and Kirshner (1982) for $\mu_{B}$ between 18 and $19.2 \mathrm{mag}$. The rest of the profile of $\mathrm{SP}$ is $0.17 \pm 0.13$ mag brighter than the 
TABLE 1

Details of Photographic Observations

\begin{tabular}{ccccc}
\hline \hline $\begin{array}{c}\text { Plate } \\
\text { Number }\end{array}$ & Date & $\begin{array}{c}\text { Exposure } \\
(\mathrm{min})\end{array}$ & Emulsion & Filter \\
\hline $104 \ldots \ldots \ldots \ldots$ & 1982 May 20 & 120 & IIa-O baked & GG 385 \\
$105 \ldots \ldots \ldots \ldots$. & 1982 May 20 & 120 & IIa-D baked & GG 495 \\
$453 \ldots \ldots \ldots \ldots$. & 1984 Apr 27 & 10 & 103a-O baked & GG 385 \\
$454 \ldots \ldots \ldots \ldots$. & 1984 Apr 27 & 10 & 103a-D baked & GG 495 \\
$462 \ldots \ldots \ldots \ldots$. & 1984 May 28 & 30 & 103a-O baked & GG 385 \\
\hline
\end{tabular}

one of Boroson, probably because of a calibration difference. There seems to be still a small systematic difference that we believe would not affect significantly the results obtained by this work. The position angle and axial ratio obtained by SP are in good agreement with those obtained by Boroson, and the integrated magnitude $B_{T}$ agrees with the value in RC2.

\section{RESULTS}

The $B$ isophotal map, with steps between contours of 0.5 mag, is presented in Figure 2. The mean $B$ and $V$ luminosity distributions are listed in Table 2 , together with the $B-V$ distribution.

Numerical integration of intensity $I(x, y)$ over the final image of the galaxy has led to the values 13.14 for the $B$ magnitude and 12.33 for the $V$. In order to obtain the total magnitude we integrated to infinite radius the contributions to the total luminosity of the bulge and disk components that we have detected in the mean luminosity profile (as described in the following sections). The contributions of the other components were also considered, as explained at the end of $\S \mathrm{V}$. The total color index $(B-V)_{T}=0.82$ was assumed to be the asymptotic value obtained from the $B$ and $V$ luminosity distributions. After correction for galactic absorption, internal absorption, and redshift (RC2), we obtain $(B-V)_{T}^{0}=0.68$. The magnitudes are listed in Table 3.

The distance modulus was derived from the galactocentric radial velocity of $3308 \mathrm{~km} \mathrm{~s}^{-1}$ (Martin 1976). Adopting $H=100 \mathrm{~km} \mathrm{~s}^{-1} \mathrm{Mpc}^{-1}$, we obtain the value 32.60 .
In order to derive the inclination $i$ of the galaxy, ellipses were fitted to outer $B$ and $V$ isophotes and a mean axial ratio was obtained. We have assumed that the disk of the galaxy can be represented by an oblate spheroid of intrinsic axial ratio equal to 0.23 (Binney and de Vaucouleurs 1981). The directions of the minor and major axes of the galaxy were defined as the mean value from the directions of the axes of the fitted ellipses. The total diameters were defined as the diameters of the last $B$ isophote in the directions obtained. The position angle is defined as the angle between the north direction and the major axis, measured eastward.

The above parameters, as well as photometric parameters at half of the total luminosity of the galaxy (de Vaucouleurs 1962), are listed in Table 3.

\section{MORPHOLOGY}

From an analysis of the short-, intermediate- and longexposure plates, we can distinguish in the central region a very bright stellar nucleus (Fig. $3 a, 4 a$ ) and a diffuse and asymmetric bar, brighter in the southeast direction (Fig. $3 b$ ). Another structure seems also to be present in the long-exposure plates (Figs. $3 c$ and $4 b$ ), which could be an internal lens. One of the spiral arms starts from this structure, while the other seems to be disconnected from the central region. This fact represents a peculiarity in barred systems, where, in general, the arms start from the bar or from an internal ring. Numerous $\mathrm{H}$ II regions over the arms are observed in the long-exposure $B$ plate.

In order to make a more quantitative study of the above subsystems, ellipses were fitted to all the $B$ and $V$ isophotes. The values obtained for the semimajor axis, semiminor axis, axial ratio, and orientation of the major axis, measured from the north eastward, are listed in Table 4 . From this table, we conclude: (1) the nucleus has a mean radius of $\sim 3 \prime 2 ;$ (2) the bar has a semimajor axis of $10^{\prime \prime}$, a semiminor axis of $7^{\prime \prime}$, and a mean axial ratio of 0.66 ; $(3)$ the disk region has a mean axial ratio of 0.86 ; (4) the lens seems to appear in the $V$ isophote at $\mu=20.81$ with major and minor half diameters of 14.5 and 10 ". 4 , respectively, corresponding to an axial ratio of 0.72 .

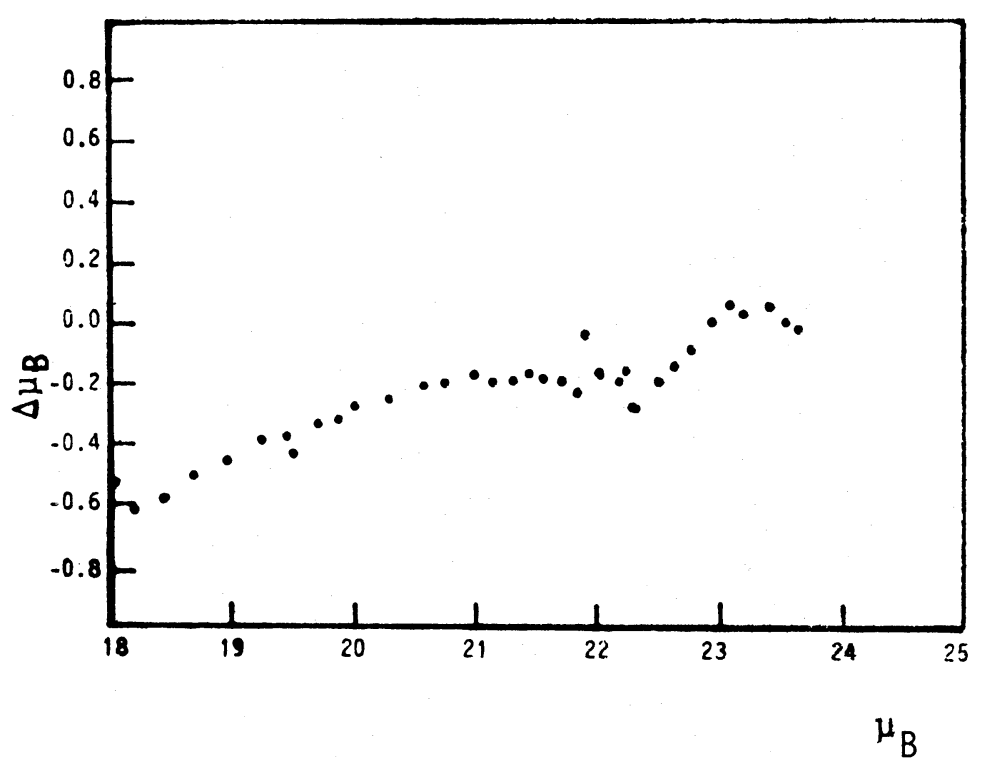

FIG. 1.-Difference between the average profile obtained for NGC 2855 and the one of Boroson 


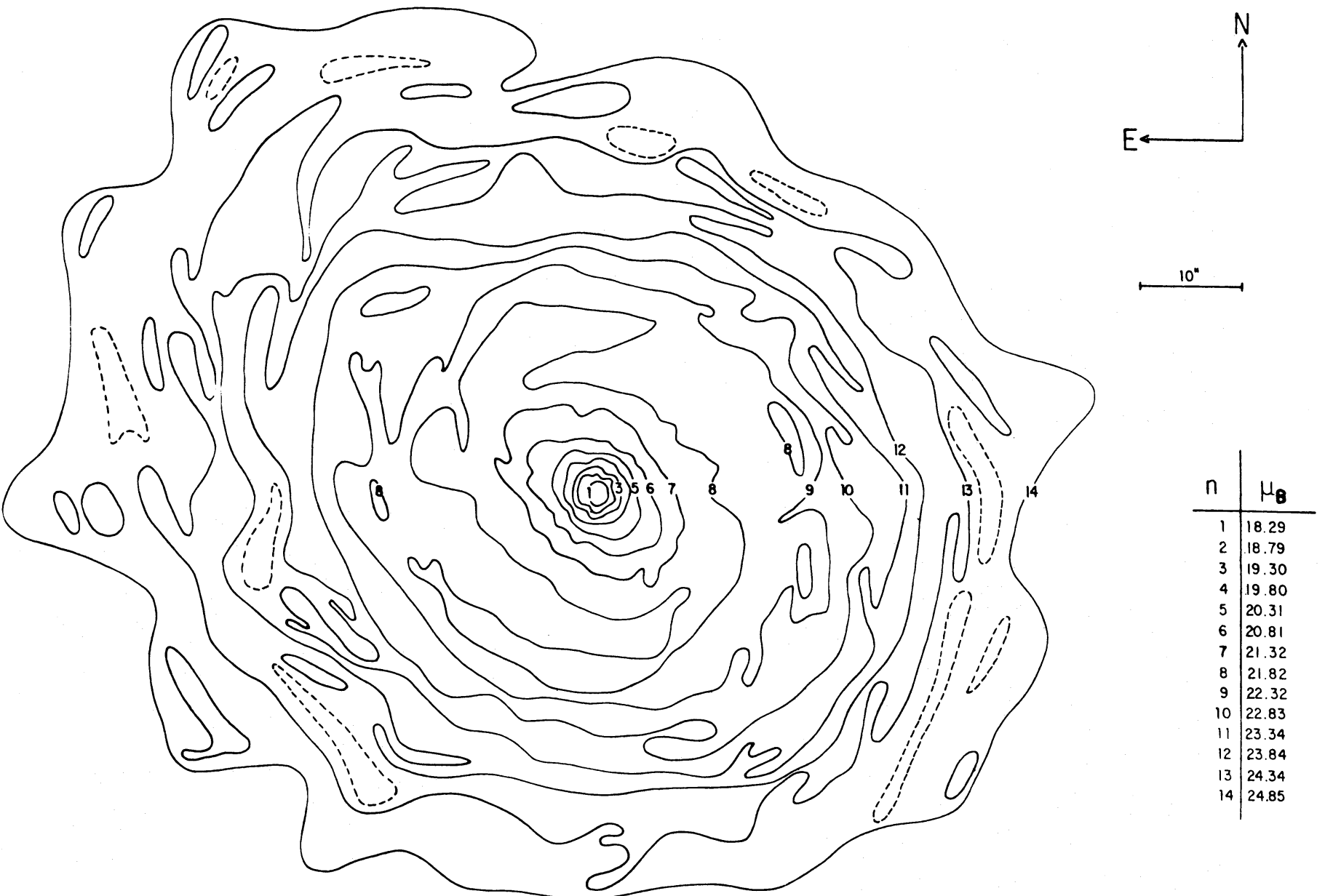

FIG. 2. $-B$ isophote map

TABLE 2

Mean $B$ and $V$ Luminosity Distributions

\begin{tabular}{ccccc}
\hline \hline$r^{* \mathrm{a}}$ & $K_{B}\left(r^{*}\right)^{\mathrm{b}}$ & $K_{V}\left(r^{*}\right)^{\mathrm{b}}$ & $B-V\left(r^{*}\right)^{\mathrm{c}}$ & $(B-V)_{T}^{\mathrm{d}}$ \\
\hline $3 \ldots \ldots$ & 0.127 & 0.144 & 0.956 & 0.956 \\
$6 \ldots \ldots$ & 0.217 & 0.260 & 1.084 & 1.011 \\
$9 \ldots \ldots$ & 0.291 & 0.340 & 0.911 & 0.987 \\
$12 \ldots \ldots$ & 0.365 & 0.411 & 0.785 & 0.949 \\
$15 \ldots \ldots$ & 0.438 & 0.486 & 0.829 & 0.930 \\
$18 \ldots \ldots$ & 0.515 & 0.560 & 0.782 & 0.909 \\
$21 \ldots \ldots$ & 0.593 & 0.628 & 0.673 & 0.881 \\
$24 \ldots \ldots$ & 0.661 & 0.691 & 0.739 & 0.867 \\
$27 \ldots \ldots$ & 0.721 & 0.739 & 0.561 & 0.845 \\
$30 \ldots \ldots$ & 0.768 & 0.775 & 0.543 & 0.828 \\
$33 \ldots \ldots$. & 0.804 & 0.803 & 0.546 & 0.817 \\
$36 \ldots \ldots$ & 0.827 & 0.827 & 0.838 & 0.818 \\
$39 \ldots \ldots$ & 0.850 & $\ldots$ & $\ldots$ & $\ldots$ \\
$42 \ldots \ldots$ & 0.867 & $\ldots$ & $\ldots$ & $\ldots$ \\
$45 \ldots \ldots$ & 0.884 & $\ldots$ & $\ldots$ & $\ldots$ \\
$\infty \ldots \ldots$ & 1.000 & 1.000 & $\ldots$ & 0.818 \\
\hline
\end{tabular}

${ }^{\text {a }}$ Equivalent radius in arc seconds.

${ }^{\mathrm{b}}$ Fraction $B$ and $V$ luminosity enclosed within the isophote with equivalent radius $r^{*}$.

c Value for the shell with outer radius $r^{*}$ and inner radius the previous one.

${ }^{\mathrm{d}}$ Integrated value, from the center.
The spiral arms appear at $\sim 17^{\prime \prime}$ from the nucleus when the distance is measured in the minor axis direction, or $\sim 20^{\prime \prime}$ after correction to face on position.

\section{LUMINOSITY PROFILES}

Radial luminosity profiles, spaced $10^{\circ}$ in angle, were obtained in the plane of the sky in order to analyze the lumin-

TABLE 3

Global Photometric Parameters

\begin{tabular}{|c|c|c|}
\hline Parameter & $B$ & $V$ \\
\hline Integrated magnitude $\ldots \ldots \ldots \ldots \ldots \ldots \ldots \ldots$ & 12.92 & 12.10 \\
\hline Corrected integrated magnitude & 12.39 & 11.71 \\
\hline Distance $(\mathrm{Mpc}) \ldots \ldots \ldots \ldots \ldots \ldots$ & 33.08 & 33.08 \\
\hline Corrected absolute magnitude ............... & -20.21 & -20.89 \\
\hline Mean axial ratio $b / a \ldots \ldots$ & 0.86 & 0.86 \\
\hline Inclination ............ & $31: 6$ & 31.6 \\
\hline \multicolumn{3}{|l|}{ Half-diameter $a$ at $\mu_{B}=24.85$ : } \\
\hline In arc seconds $\ldots \ldots \ldots \ldots \ldots$ & 53.3 & $\ldots$ \\
\hline In $\mathrm{kpc} \ldots \ldots \ldots \ldots \ldots \ldots \ldots$ & 8.55 & $\ldots$ \\
\hline Position angle $\ldots \ldots \ldots \ldots \ldots \ldots$ & 61.7 & $61: 7$ \\
\hline Equivalent effective radius $r_{e}^{*} \ldots \ldots \ldots \ldots \ldots$ & $17 " .3$ & $15^{\prime \prime} 8$ \\
\hline Effective surface brightness ... & 21.95 & 21.10 \\
\hline \multicolumn{3}{|l|}{ Concentration indices: $:^{\mathrm{a}}$} \\
\hline $\mathrm{C}_{21}=r_{e}^{*} / r_{1}^{*} \ldots \ldots \ldots \ldots \ldots \ldots \ldots \ldots \ldots \ldots \ldots \ldots \ldots$ & 2.31 & 2.87 \\
\hline $\mathrm{C}_{32}=r_{3}^{*} / r_{e}^{*} \ldots \ldots \ldots \ldots \ldots \ldots \ldots \ldots \ldots \ldots \ldots \ldots \ldots \ldots \ldots$ & 1.68 & 1.76 \\
\hline
\end{tabular}

${ }^{a}$ The quantities $r_{e}^{*}, r_{1}^{*}$ and $r_{3}^{*}$ are defined by $K\left(r_{e}^{*}\right)=\frac{1}{2}, K\left(r_{1}^{*}\right)=\frac{1}{4}$, $K\left(r_{3}^{*}\right)=\frac{3}{4}$ 

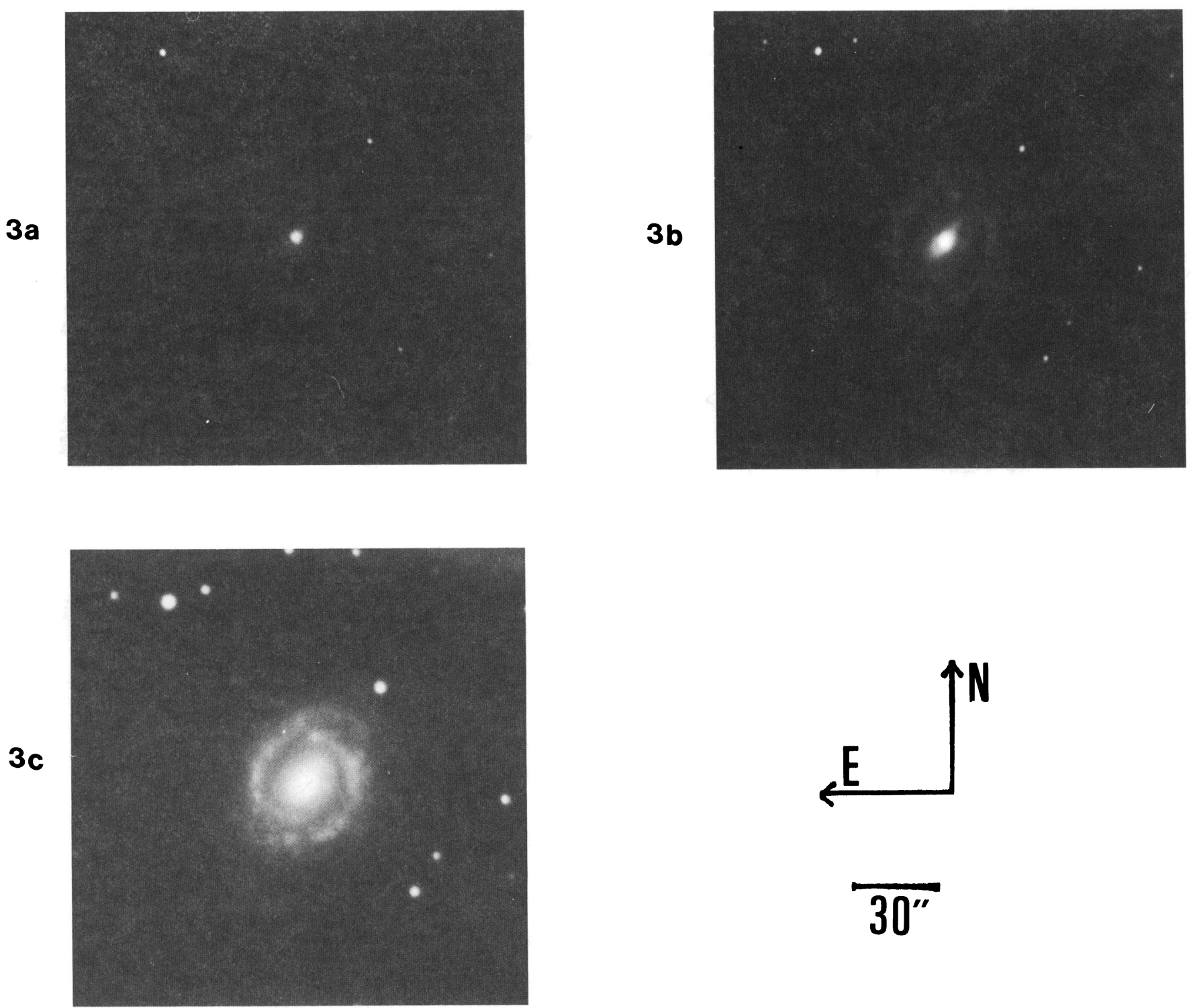

FIG. 3
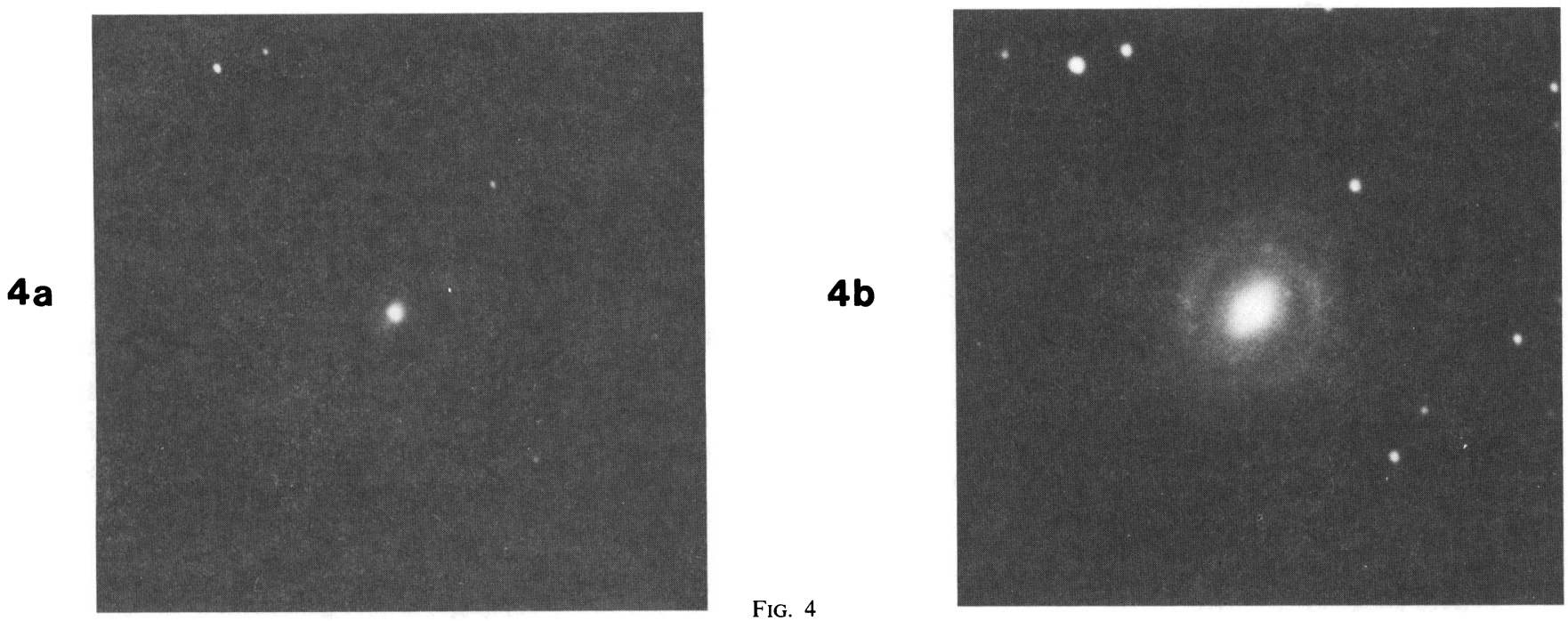

FIG. 3. $-(a)$ Short-exposure $B$ plate; $(b)$ intermediate-exposure $B$ plate; $(c)$ long-exposure $B$ plate Fig. 4.-(a) Short-exposure $V$ plate; $(b)$ long-exposure $V$ plate

(C) American Astronomical Society - Provided by the NASA Astrophysics Data System 
TABLE 4

Parameters of Ellipses FitTed to $B$ AND $V$ Isophotes

\begin{tabular}{cccccccc}
$\mu_{B}$ & $\mu_{V}$ & $\theta_{B}{ }^{\mathrm{a}}$ & $\theta_{V}$ & $a_{B}$ & $a_{V}$ & $b / a_{B}$ & $b / a_{V}$ \\
\hline $18.79 \ldots \ldots$ & 17.78 & 90.1 & 65.7 & 1.9 & 1.6 & 0.90 & 0.91 \\
$19.30 \ldots \ldots$ & 18.29 & 78.3 & 67.6 & 2.5 & 2.5 & 0.90 & 0.91 \\
$19.80 \ldots \ldots$ & 18.79 & 55.3 & 40.9 & 3.3 & 3.8 & 0.93 & 0.83 \\
$20.31 \ldots \ldots$ & 19.30 & 47.7 & 50.0 & 5.0 & 5.7 & 0.76 & 0.72 \\
$20.81 \ldots \ldots$ & 19.80 & 51.9 & 50.4 & 7.7 & 8.0 & 0.64 & 0.64 \\
$21.32 \ldots \ldots$ & 20.31 & 47.2 & 48.8 & 10.7 & 10.1 & 0.68 & 0.68 \\
& 20.81 & $\ldots$ & 47.1 & $\ldots$ & 14.5 & $\ldots$ & 0.72 \\
$21.82^{\mathrm{b}} \ldots \ldots$ & $21.32^{\mathrm{b}}$ & 57.1 & 23.1 & 17.9 & 20.7 & 0.80 & 0.92 \\
$22.33 \ldots \ldots$ & 21.82 & 65.9 & 58.5 & 25.2 & 26.7 & 0.89 & 0.90 \\
$22.83 \ldots \ldots$ & 22.33 & 62.6 & 63.4 & 30.6 & 31.4 & 0.83 & 0.85 \\
$23.34 \ldots \ldots$ & 22.83 & 63.9 & 66.4 & 35.4 & 35.1 & 0.79 & 0.89 \\
$23.84 \ldots \ldots$ & 23.34 & 61.6 & 52.7 & 38.0 & 39.5 & 0.85 & 0.89 \\
$24.34 \ldots \ldots$ & $\ldots$ & 61.1 & $\ldots$ & 43.3 & $\ldots$ & 0.87 & $\ldots$ \\
$24.85 \ldots \ldots$ & $\ldots$ & 60.9 & $\ldots$ & 51.6 & $\ldots$ & 0.84 & $\ldots$
\end{tabular}

a Angle, in degrees, between north and major axis direction, measured eastward.

b Spiral arms distort this isophotal level. osity distribution of the components and, in particular, the behavior of the color index $B-V$. Figure 5 shows the $B$ and $V$ profiles along the major and minor axes of the galaxy.

In order to separate the contribution of the different components to the total luminosity of the galaxy, "elliptically averaged" (Boroson 1981) profiles were obtained. To do this, ellipses, centered on the nucleus, were fitted to outer isophotes, and the apparent flattening and position angles of the major axes were obtained. Then the profiles were coadded, each one being stretched according to the radius of the ellipses at that angle relative to the major axis.

A tentative least-squares fitting to the average $B$ luminosity profile was made using the method developed by Kormendy (1977), which fits a de Vaucouleurs (1962) curve for the bulge, $\mu=\mu_{e}+8.325\left[\left(r / r_{e}\right)^{1 / 4}-1\right]$, and an exponential curve for the disk, $\mu=\mu_{0}+1.0857 \alpha r$ (Freeman 1970). The surface brightness of the disk region was fitted first, within the diskdominated range. This range was defined as that part of the profile beyond the prominent spiral feature, which obscures the

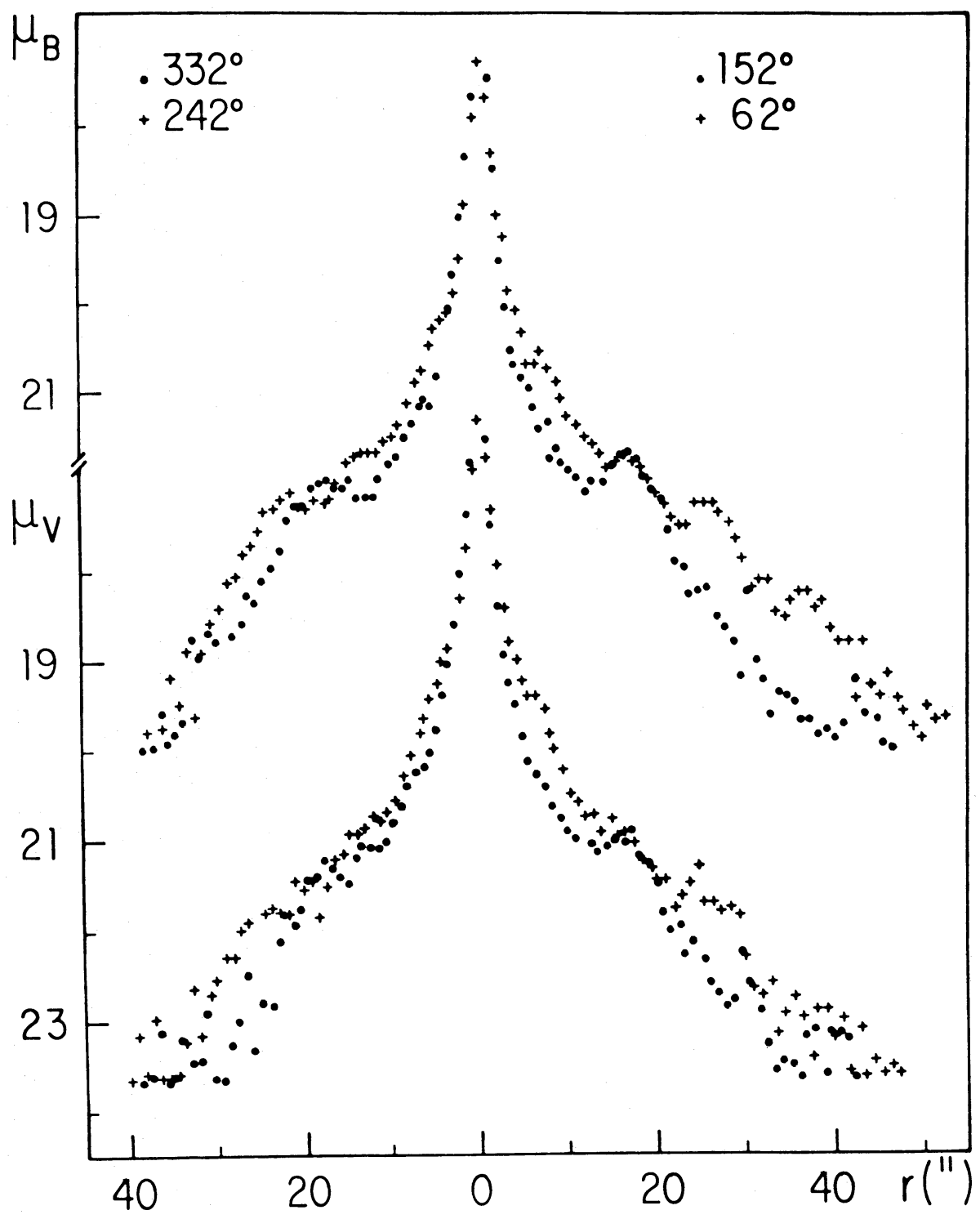

FIG. 5. $-B$ and $V$ luminosity profiles along major $(+)$ and minor $(\bullet)$ axis 
underlying internal part of the disk. Since that range extends over only $1 \mathrm{mag}$ in intensity, the disk parameters should be taken with caution. This calculated disk contribution was then subtracted from the observed data points at all radii, and to these corrected data the bulge function was fitted in the range dominated by the bulge light. This fit was subtracted from the original observed profile, and the process was repeated until it converged. For the average $V$ profile it was not possible to define a disk-dominated range, and only the de Vaucouleurs function was fitted to the nuclear region. The average profiles and fitted functions are shown in Figure 6.

The parameters $\mu_{e}, r_{e}, \mu_{0}$, and $\alpha^{-1}$ of the adjusted fitting functions, as well as the fitting ranges for each function, are listed in Table 5.

In order to calculate the contribution of each component to the $B$ total luminosity, we have integrated the exponential and $r^{1 / 4}$ law over a circular surface of radius equal to the semimajor axis of the last isophote. The contribution of the bar, lens, and spiral arms was calculated as the difference between the total

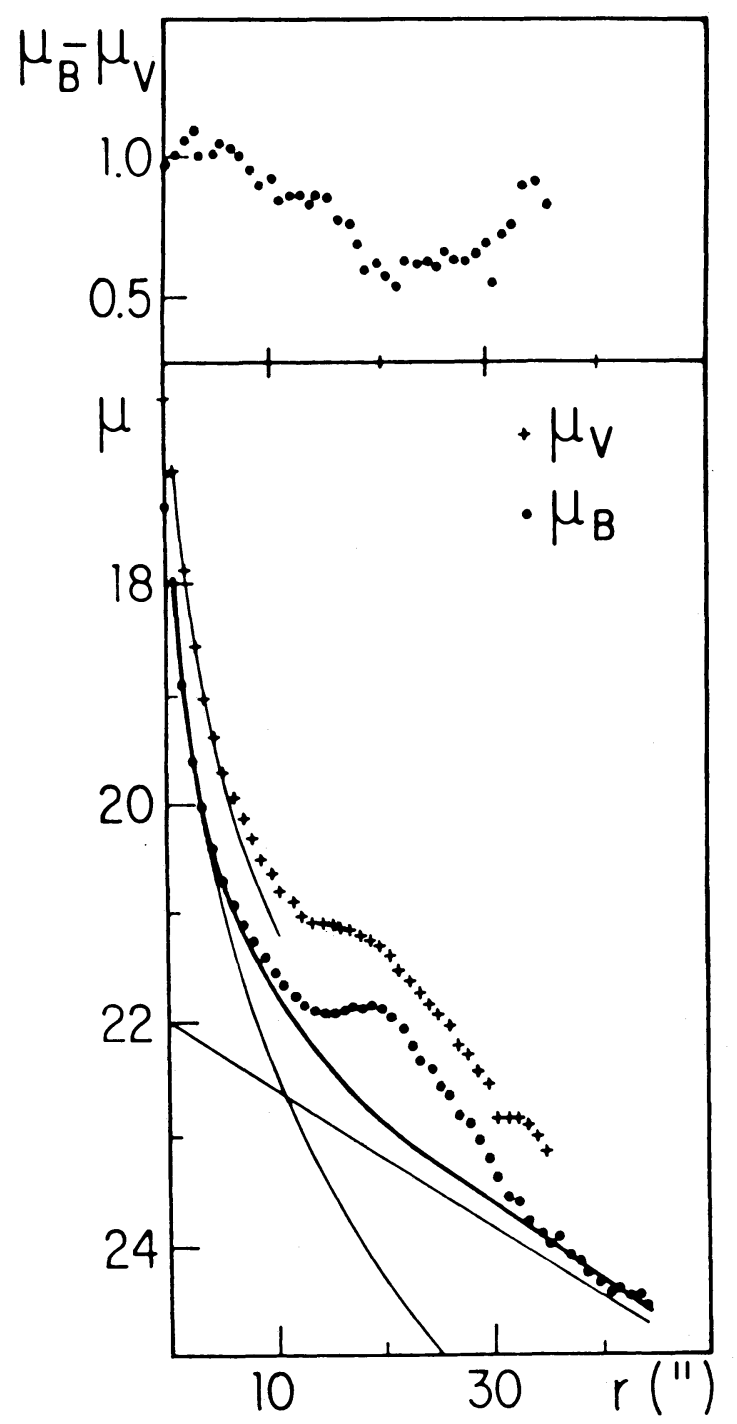

Fig. 6.-Average $B$ and $V$ luminosity profiles and fitted functions to the bulge and disk (light lines); heavy line is the sum of the two functions. On the top: average $B-V$ distribution. luminosity inside the last isophote and the sum of the contributions of the bulge and disk. Then we integrated the bulge and disk contributions to infinite radius. Assuming that the bar, lens, and spiral arms contributions do not extend beyond the last isophote, we summed the calculated contributions to obtain the value for the total luminosity. The fraction of total luminosity corresponding to each component as well as the bulge to disk ratio are listed in Table 5 .

\section{COLOR INDICES}

From the average $B$ and $V$ profiles an average $B-V$ distribution was obtained, which is shown in Figure 6. We can observe a pronounced color gradient from the nucleus, which has a mean color of $1.0 \mathrm{mag}$, to the arms, with $B-V=0.60$. This color remains constant until a radius $30^{\prime \prime}$, where it starts to become redder because of the contribution of the disk, reaching $B-V=0.85$. It is interesting to note that in the region corresponding to the lens $\left(10^{\prime \prime}-15^{\prime \prime}\right)$, the color remains constant at a value $B-V=0.85$, equal to that of the disk.

In order to make a more detailed analysis of the behavior of the $B-V$ color index over the entire galaxy we have made a schematic representation (Fig. 7).

The $B-V$ values shown inside the nuclear and bar regions were obtained by integration of the luminosity distributions. In outer regions, we have made local means, centered in the points marked in the figure and whose corresponding $B-V$ values are shown. There are three elliptical regions represented: the lens region, the most luminous part of the arms region, and an external region with major half diameter equal to 27.5 . The $B-V$ values farther than $30^{\prime \prime}$ (major axis of the external elipse) were not presented because they show too much fluctuation. It is interesting to note that, independently of the mean gradient previously described as being due to the distribution of the different components, the $B-V$ values in the southern half of the galaxy are systematically redder than those of the northern part. The effect amounts to $E(B-V)$ with a value of $0.2-0.3$.

\section{DISCUSSION}

NGC 4507 is a luminous spiral galaxy classified as SBab(rs) I by Sandage and Brucato (1979), with absolute magnitude $M_{B}=-20.21$, in agreement with the luminosity classification I. The dimensions for the major and minor half diameter at the isophotal level $\mu_{B}=24.85$ are, respectively, $8.55 \mathrm{kpc}$ and $6.47 \mathrm{kpc}$. The corrected integrated color index $(B-V)_{T}^{0}=0.68$ is normal for a galaxy of type ab.

TABLE 5

Parameters of Fitted Functions to Mean Profiles

\begin{tabular}{|c|c|c|}
\hline Parameter & $B$ & $V$ \\
\hline Bulge fitting range (arcsec) & $0.9-3.6$ & $0.9-3.6$ \\
\hline$\mu_{e} \ldots \ldots \ldots \ldots \ldots \ldots \ldots \ldots \ldots$ & 20.90 & 20.25 \\
\hline$r_{e}(\operatorname{arcsec}-\mathrm{kpc}) \ldots \ldots \ldots \ldots \ldots \ldots \ldots$ & $5.02-0.82$ & $6.75-1.11$ \\
\hline Bulge luminosity $\ldots \ldots \ldots \ldots \ldots \ldots$ & $0.37 L_{T}{ }^{a}$ & $\ldots$ \\
\hline Disk-fitting range (arc sec) & $35-45$ & $\cdot$ \\
\hline$\mu_{0} \ldots \ldots \ldots \ldots \ldots \ldots$ & 21.98 & $\ldots$ \\
\hline$\alpha^{-1}(\operatorname{arcsec}-\mathrm{kpc}) \ldots \ldots \ldots \ldots \ldots \ldots$ & $17.54-2.88$ & $\ldots$ \\
\hline Disk luminosity $\ldots \ldots \ldots \ldots \ldots \ldots \ldots$ & $0.46 L_{T}$ & $\ldots$ \\
\hline Bulge/disk $\ldots \ldots \ldots \ldots \ldots \ldots \ldots \ldots$ & 0.80 & $\ldots$ \\
\hline Bar + lens + arms $\ldots \ldots \ldots \ldots \ldots \ldots$ & $0.17 L_{T}$ & $\ldots$ \\
\hline
\end{tabular}

${ }^{\text {a }} L_{T}$ : total luminosity. 


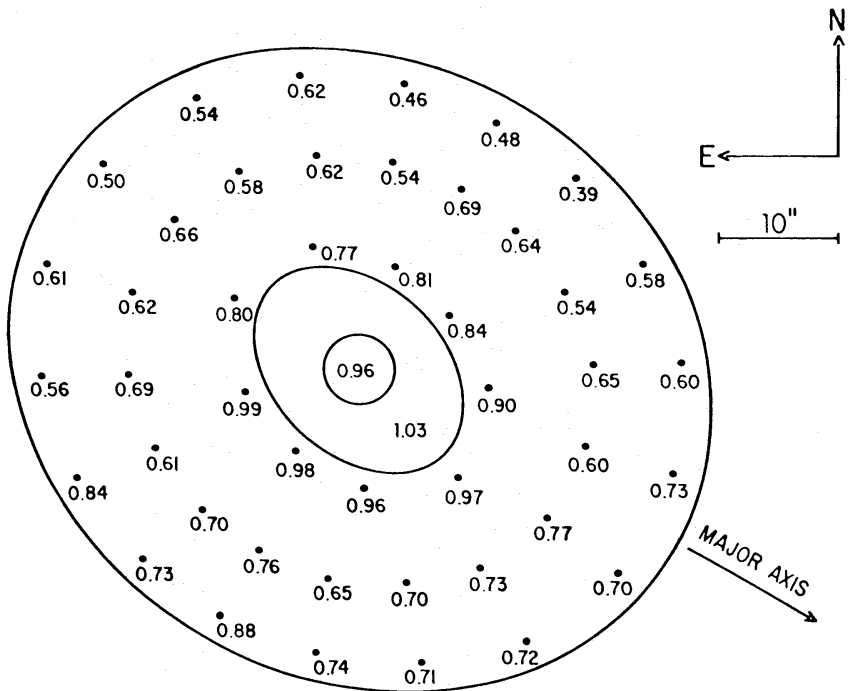

FIG. 7. $-B-V$ local values; integrated values for nucleus and bar

From the decomposition of the average luminosity profile we conclude that the galaxy has (1) a nuclear bulge obeying a $r^{1 / 4}$ law which contributes $37 \%$ of the total luminosity; (2) an a exponential disk contributing $46 \%$ of the total luminosity; (3) two well-developed spiral arms; (4) an asymmetric bar, brightest in the SE direction, with major and minor diameters of, respectively, $3.5 \mathrm{kpc}$ and $2.4 \mathrm{kpc}$; and (5) probably an internal lens, with major diameter of $4.75 \mathrm{kpc}$ and minor diameter of $3.41 \mathrm{kpc}$. These three last components together contribute $17 \%$ of the luminosity. The resulting bulge-to-disk ratio of 0.80 obeys the correlation between $B / D$ ratios and $T$ types (RC2) found by Boroson (1981) if we adopt $T=2$ for this galaxy, in accordance with the type ab. The orientation of the major axis of the lens is the same as that of the bar. The orientation of the major axis of the disk region is $\sim 10^{\circ}$ eastward from that of the bar.

If we correct our value for the central surface brightness of the disk, for galactic absorption and inclination we obtain $\mu_{0 c}=21.71$, which is similar to the value obtained by Boroson (1981) for the brightest spiral galaxies. In Figure 11 of Boroson the locus occupied by NGC 4507 is in the region of superposition of points representing $\mathrm{S} 0$ galaxies and spirals. The value for $\mu_{0 c}$ is very near the mean value $\mu_{0 c}=21.65 \pm 0.30$ obtained by Freeman (1970). The scale length $\alpha^{-1}=2.88 \mathrm{kpc}$ agrees with the values of other galaxies of the same type.

The color-index distribution shows two kinds of gradients. The first is due to the distribution of the different populations in the galaxy: the nucleus and bar have a color index of $\sim 1.00$, while the arms have $B-V \approx 0.60$; the mean color of the lens seems to be equal to that of the disk, with a value of 0.85 . The second gradient can be detected if we compare the $B-V$ values of the southern part with that of the northern part of the galaxy. These last ones are systematically bluer, probably indicating that the northwest part of the galaxy is the one inclined toward us.

The authors are grateful to C. Rité, R. R. Carvalho, P. S. Pellegrini, and L. A. N. da Costa of Observatório Nacional for assistance with the utilization of the PDS microdensitometer and reduction software, and to the Brazilian Institutions $\mathrm{CNPq}$ and FINEP for financial support.

\section{REFERENCES}

Binney, J., and de Vaucouleurs, G. 1981, M.N.R.A.S., 194, 679.

Boroson, T. 1981, Ap. J. Suppl., 46, 177.

Da Costa, L. A. N., Carvalho, R. R., Rité, C. P., and Pellegrini, P. S. S. 1982, Image Processing at ONI-Surface Photometry, Pub. Observatório Nacional, No. 2.

de Vaucouleurs, G. 1962, in IAU Symp. 15, Problems of Extragalactic Research, ed. G. C. McVittie (New York: Macmillan), p. 3.

de Vaucouleurs, G., de Vaucouleurs, A., and Corwin, H. H. 1976, Second Reference Catalogue of Bright Galaxies (Austin: University of Texas Press) (RC2).

Freemann, K. C. $1970, A p . J, 160,811$

Griersmith, D. 1980, A. J., 85, 789 .

Kormendy, J. 1977, Ap. J., 217, 406

Martin, W. L. 1976, M.N.R.A.S., 175, 633

Sandage, A., and Brucato, R. 1979, A.J., 84, 472.

Schröder, M. F. S., and Pastoriza, M. G. 1986, Ap. J., submitted.

Wegner, G. 1979, Ap. Space Sci., 60, 15.

Whitmore, B. C., and Kirshner, R. P. 1982, A.J., 87, 500.

Thaisa Storchi Bergmann and Miriani Griselda Pastoriza: Instituto de Física, Universidade Federal do Rio Grande do Sul, Av. Bento Gonçalves, 9500, 90049-Porto Alegre, RS, Brazil 